\title{
Glimpses of the Lichen Flora of Achanakmar-Amarkantak Biosphere Reserve in Central India
}

\author{
Achuta Nand Shukla ${ }^{1}$, Krishna Pal Singh ${ }^{2}$ \\ ${ }^{1}$ Botanical Survey of India, Northern Regional Centre, Dehradun, India; ${ }^{2}$ Botanical Survey of India, Central Regional Centre, Alla- \\ habad, India. \\ Email: achutbsi@gmail.com
}

Received April 5 $5^{\text {th }}$ 2012; revised May 12 ${ }^{\text {th }}, 2012$; accepted June $2^{\text {nd }}, 2012$

\begin{abstract}
The present paper enumerates an account of the lichen flora in Achanakmar-Amarkantak Biosphere Reserve located in Central India which comprises 78 species belonging to 25 genera under nine families. Of these, twenty two species have been reported for the first time from Central India. All the species are enumerated with their correct original citation, basionyms if any, their places of occurrence in the reserve area, distributional range and exsiccata have been provided.
\end{abstract}

Keywords: Lichen; Contributions; Achanakmar-Amarkantak; Central India

\section{Introduction}

India, being one of the megadiversity countries in the world, has designated 16 Biosphere Reserves all over the country [1]. Of these, Pachmarhi Biosphere Reserve and Achanakmar-Amarkantak Biosphere Reserve (AABR) are located in Central India. Achanakmar-Amarkantak Biosphere Reserve an interstate biosphere reserve situated in Madhya Pradesh and Chhattisgarh states (Figure 1).

The Achanakmar-Amarkantak Biosphere Reserve (AABR) comprising of an area $3835.51 \mathrm{~km}^{2}$, lies between $22^{\circ} 15^{\prime} \mathrm{N}$ to $22^{\circ} 58^{\prime} \mathrm{N}$ lat. and $81^{\circ} 25^{\prime} \mathrm{E}$ to $82^{\circ} 50^{\prime} \mathrm{E}$ long. in the states of Madhya Pradesh and Chhattishgarh. From the existing demarcated area of the reserve, $c a 551.15 \mathrm{~km}^{2}$ of area was earlier marked as Achanakmar Wild Life Sanctuary has now been designated as core zone of this reserve and remaining area of $3284.36 \mathrm{~km}^{2}$ is marked as buffer zone (Figure 1).

Of the total area of buffer zone, ca $1224.98 \mathrm{~km}^{2}$ falls in Madhya Pradesh and remaining area of $2059.38 \mathrm{~km}^{2}$ in Chhattisgarh states [2]. The biosphere reserve area is one of major watersheds of peninsular India separating rivers draining into Arabian Sea and Bay of Bengal. The reserve is the source of three major river systems viz., Narmada, Johilla and Sone. The area has a typical monsoon climate with three distinct seasons viz., summer from March to June, rainy season from July to October and winter season from November to February. The reserve has the varied topography and climatic conditions which provide congenial habitat for the unique diversity of plants. The vegetation of the forest area of the reserve represents tropical deciduous and can be further classified into Northern Tropical Moist Deciduous and Southern Dry Mixed Deciduous forests [3]. The altitudinal ranges of reserve with varied climatic and edaphic conditions give rise to rich and luxuriant vegetation in Central India [4-7]. During the survey it has been noticed that the area has several intermittent patches of moist and dry deciduous forest. Shorea robusta is the major component of these forests, associated with the tree species of Terminalia and Syzygium. It is interesting to note that sal forest provides a most congenial habitat for lichen growth as they are exposed to rain, sunlight and wind currents. Majority of the lichens belong to foliose forms show their predominance as the species of Parmotrema and Dirinaria are found growing commonly on young branches and old tree trunk having hard bark at breast height. The species of Lecanora shows luxuriant growth on soft and smooth trunk of young sal tree. Among these, the species of Pertusaria show their rich growth on sal tree particularly growing in dense moist places along the streams. In addition to these, other taxa of lichen are also abundant in humid and shady areas of the sal forest. These taxa are Buellia, Lecanora, Canoparmelia, Bulbothrix, etc.

The study of literature reveals that not much work has been done on the lichen flora of Achanakmar-Amarkantak Biosphere Reserve except Nayaka et al. [8] and Upreti et al. [9] in which they have reported 32 species belonging to 22 genera and 26 families from Achanakmar Wildlife Sanctuary. The present report of 78 species of lichen are the additions to the earlier record made by Nayaka et al. [8]. 


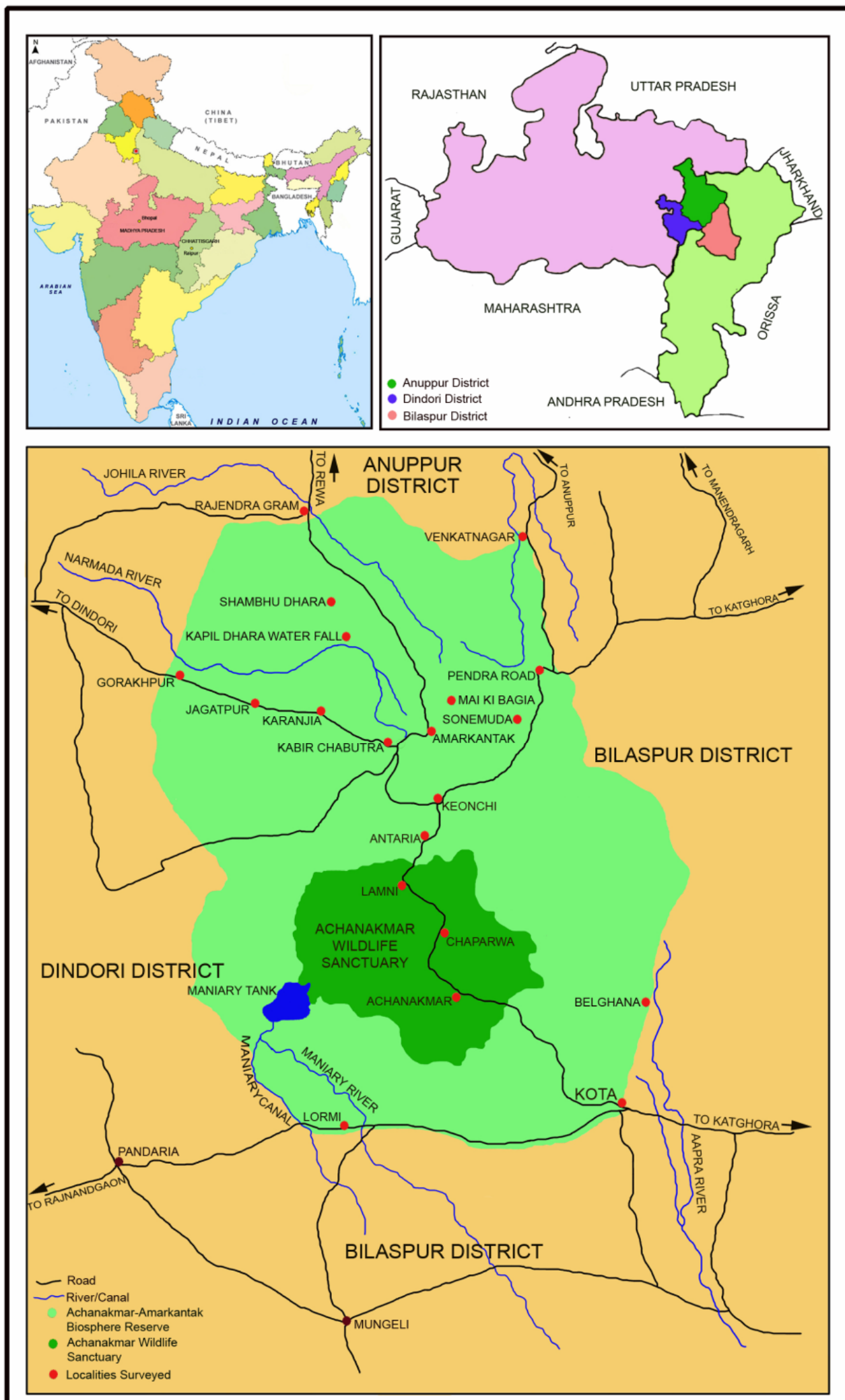

Map: Achanakmar-Amarkantak Biosphere Reserve showing explored localities and its location within India, Madhya Pradesh and Chhattisgarh

Figure 1. Map of Achanakmar-Amarkantak Biosphere Reserve, Central India. 
During an intensive and extensive survey in year 20042008 the authors have collected 78 species belonging to 25 genera and nine families from the AchanakmarAmarkantak Biosphere Reserve area. Of these, twenty two species viz., Arthothelium chiodectoides (Nyl.) Zahlbr., Arthothelium saxicolum Makhija \& Patw., Amandinea diorista (Nyl.) Marbach, Hafellia disciformis (Fr.) Mar bach \& H.Mayrhofer, Bulbothrix setschwanensis (Zahlbr.) Hale, Caloplaca vitellinula (Nyl.) H.Oliver, Canoparmelia aptata (Kremp.) Elix \& Hale, Canoparmelia cinerascens (Lynge) Elix \& Hale, Canoparmelia crozalsiana (de Lesd.) Elix \& Hale, Canoparmelia eruptens (Kurok.) Elix \& Hale, Heterodermia albidiflava (Kurok.) D. D. Awasthi, Heterodermia angustiloba (Müll. Arg.) D. D. Awasthi, Heterodermia dactyliza (Nyl.) Swinscow \& Krog, Heterodermia dissecta (Kurok.) D. D. Awasthi, Heterodermia tremulans (Müll.Arg.) W.L.Culb., Hypotrachyna awasthii Hale \& Patw., Hypotrachyna crenata (Kurok.) Hale, Lecanora argentata (Ach.) Degel., Lecanora varia (Hoffm.) Ach., Leptogium asiaticum M. Jørg., Pertusaria concinna Erichsen, Physcia tribacia (Ach.) Nyl. are reported for the first time from the Central India.

\section{Methodology}

The lichens were collected in 2004 to 2008 from various localities of Achanakmar-Amarkantak Biosphere Reserve viz., Sonemuda, Kapildhara, Kabirchabutara, Doodhdhara, Sambhudhara, Tikaritola, Karanjia, Jagatpur, Achanakmar etc. All the specimens have been deposited in the herbarium at Botanical Survey of India, Central Regional Centre, Allahabad (BSA).

All the 78 species have been enumerated with the correct nomenclatural citation, basionym if any, the exact locality of it occurrence in the reserve area, distributional range and exsiccata.

\section{Enumeration}

\section{Family-Arthoniaceae}

1. Arthothelium chiodectoides (Nyl.) Zahlbr., Cat. Lich. Univ. 2: 122. 1922; D. D. Awasthi, Beih. Nova Hedwigia 17: 27. 1965. Arthonia chiodectoides Nyl., Flora 52: 72. 1869.

Ecology and Distribution: On tree bark, Sambhudhara, $1002 \mathrm{~m}$.

Range of Distribution: India (Goa, Himachal Pradesh, Karnataka, Maharashtra and West Bengal, Madhya Pradesh).

Specimen examined: Sambhudhara, A. N. Shukla 1495 (BSA), det. A. N. Shukla.

2. Arthothelium saxicolum Makhija \& Patw., Lichenologist 29(2): 169. 1997.

Ecology and Distribution: On rocks, Sonemuda, Mai-
Ki-Bagia, 950 - 960 m.

Range of Distribution: India (Maharashtra, Madhya Pradesh). Endemic.

Specimens examined: Sonemuda, A. N. Shukla 1777 (BSA); Mai-Ki-Bagia, G. P. Sinha 1161 (BSA), det. A. N. Shukla.

\section{Family-Coccocarpaceae}

3. Coccocarpia palmicola (Spreng.) Arv. \& D. J. Galloway, Bot. Not. 132: 242. 1979; D. D. Awasthi, Kavaka 12(2): 84. 1985. Lecidea palmicola Spreng., Kongl. Vetensk. Acad. Nya Handl. 1: 46. 1820.

Ecology and Distribution: On tree bark, rocks, soils and leaves, Kabirchabutara, $1000 \mathrm{~m}$.

Range of Distribution: India (Andaman \& Nicobar Islands, Arunachal Pradesh, Assam, Karnataka, Kerala, Madhya Pradesh, Maharashtra, Manipur, Meghalaya, Nagaland, Sikkim, Tamil Nadu, Uttarakhand and West Bengal); tropical and subtropical regions of the world.

Specimen examined: Kabirchabutara, G. P. Sinha 2614 (BSA), det. A. N. Shukla.

\section{Family-Collemataceae}

4. Collema nigrescens (Huds) DC. in Lamarck \& de Candolle, Fl. Franç., ed. 3, 2: 384. 1805; D. D. Awasthi, Beih. Nova Hedwigia 17: 46. 1965. Lichen nigrescens Huds., Fl. Angl. 1762.

Ecology and Distribution: On tree bark, Kabirchabutara, $1000 \mathrm{~m}$.

Range of Distribution: India (Madhya Pradesh, Tamil Nadu and Uttarakhand), Canada, China; North Asia, Europe.

Specimen examined: Kabirchabutra, G. P. Sinha 2655 (BSA), det. A. N. Shukla.

5. Leptogium asiaticum M. Jørg., Herzogia 2: 466. 1973; D. D. Awasthi \& Akhtar, Norweg. J. Bot. 24: 63. 1977.

Ecology and Distribution: On tree bark, Dhunipani, $900 \mathrm{~m}$.

Range of Distribution: India (Madhya Pradesh, Manipur, Nagaland and Sikkim), Australia, China, Japan, Nepal, Sri Lanka; Africa.

Specimen examined: Dhunipani, A. N. Shukla 2547 (BSA), det. A. N. Shukla.

6. Leptogium austroamericanum (Malme) C. W. Dodge, Ann. Missouri Bot. Gard. 20: 419. 1933; D. D. Awasthi, Geophytology 5: 179. 1975. Leptogium cyanescens var. austroamericanum Malme, Ark. Bot. 19(8): 11. 1924.

Ecology and Distribution: On tree bark, Kabirchabutara, $1000 \mathrm{~m}$.

Range of Distribution: India (Andaman \& Nicobar Islands, Arunachal Pradesh, Goa, Himachal Pradesh, Karnataka, Kerala, Madhya Pradesh, Maharashtra, Manipur and Uttarakhand), Australia, Taiwan, Thailand; Europe, North, Central and South America.

Specimen examined: Kabirchabutara, G. P. Sinha 1175 (BSA), det. A. N. Shukla. 
7. Leptogium cyanescens (Rabenh.) Körb., Syst. Lich. Germ.: 420. 1885; D. D. Awasthi \& Akhtar, Geophytology 8(2): 195. 1979. Collema cyanescens Rabenh., Deutschl. Krypt. Fl. 2: 50. 1845.

Ecology and Distribution: On tree bark, rocks and soils, Kabirchabutra, 1000 m.

Range of Distribution: India (Arunachal Pradesh, Himachal Pradesh, Jammu \& Kashmir, Kerala, Madhya Pradesh, Maharashtra, Manipur, Nagaland, Tamil Nadu, Uttarakhand and West Bengal-hills), Australia; Africa, Asia, North, Central and South America.

Specimen examined: Kabirchabutra, G. P. Sinha 2716 (BSA), det. A. N. Shukla.

8. Leptogium denticulatum Nyl., Ann. Sci. Nat., Bot., ser. 5, 7: 302. 1867; Kr. P. Singh \& S. Singh, Geophytology 6(1): 29. 1976.

Ecology and Distribution: On tree bark, rocks and soils. Kapildhara, $1000 \mathrm{~m}$.

Range of Distribution: India (Andaman \& Nicobar Islands, Arunachal Pradesh, Goa, Karnataka, Kerala, Madhya Pradesh, Maharashtra, Manipur, Meghalaya, Nagaland, Sikkim, Tamil Nadu and West Bengal-hills), Taiwan; mainly tropical regions of America.

Specimen examined: Kapildhara, G. P. Sinha 1200 (BSA), det. A. N. Shukla.

\section{Family-Lecanoraceae}

9. Lecanora argentata (Ach.) Degel., Ark. Bot. 24 A: 78. 1931; Upreti, Feddes Repert. 108: 187. 1997. Parmelia subfusca var. Argentata Ach., Meth. Lich.: 169. 1803.

Ecology and Distribution: On tree bark, Forest medicinal garden, $1040 \mathrm{~m}$.

Range of Distribution: India (Andaman \& Nicobar Islands, Andhra Pradesh, Madhya Pradesh, Maharashtra, Manipur, Orissa, West Bengal-plains); Africa, Asia, Australsia, Europe, North America.

Specimen examined: Forest medicinal garden, A. N. Shukla 1518 (BSA), det. A. N. Shukla.

10. Lecanora coronulans Nyl., Flora 59: 510. 1876; Upreti \& Chatterjee, Feddes Repert. 108: 577. 1997.

Ecology and Distribution: On tree bark, Sambhudhara, $1002 \mathrm{~m}$.

Range of Distribution: India (Himachal Pradesh, Madhya Pradesh, Tamil Nadu and Uttarakhand); Central and South America.

Specimen examined: Sambhudhara, A. N. Shukla 2501 (BSA), det. A. N. Shukla.

11. Lecanora perplexa Brodo, Beih. Nova Hedwigia 79: 148-150. 1984; Upreti, Feddes Repert. 108: 198. 1997.

Ecology and Distribution: On tree bark, Mai-Ki-Bagia, $960 \mathrm{~m}$.

Range of Distribution: India (Arunachal Pradesh, Jammu \& Kashmir, Karnataka, Kerala, Madhya Pradesh, Orissa, Rajasthan, Tamil Nadu and Uttarakhand) and Nepal.
Specimen examined: Mai-Ki-Bagia, G. P. Sinha 1155 (BSA), det. A. N. Shukla.

12. Lecanora varia (Hoffm.) Ach., Lich. Univ.: 377. 1810; D. D. Awasthi, Beih. Nova Hedwigia 17: 65. 1965. Patellaria varia Hoffm., Descr. Pl. Cl. Crypt. 1: 102. Table 23, Figure 4. 1790.

Ecology and Distribution: On tree bark and rocks, Jagatpur, Karanjia, 850 - 910 m.

Range of Distribution: India (Madhya Pradesh, Manipur, Tamil Nadu and Uttarakhand); widely distributed in the world.

Specimens examined: Jagatpur, A. N. Shukla 2900, 2905 (BSA); Karanjia, A. N. Shukla 2898 (BSA), det. A. N. Shukla.

\section{Family-Letrouitiaceae}

13. Letrouitia domingensis (Pers.) Hafellner \& Bellem., Nova Hedwigia 35: 281. 1982; D. D. Awasthi \& Srivastava, Proc. Indian Acad. Sci., Pl. Sci. 99(3): 173. 1989. Patellaria domingensis Pers., Ann. Wetterauisch Gesammte Ges. Naturk. 2: 12. 1810.

Ecology and Distribution: On tree bark, Durgadhara, $760 \mathrm{~m}$.

Range of Distribution: India (Andhra Pradesh, Arunanchal Pradesh, Jharkhand, Karnataka, Madhya Pradesh, Orissa, Tamil Nadu and West Bengal); tropical region of the world.

Specimen examined: Durgadhara, A. N. Shukla 1520 (BSA), det. A. N. Shukla.

\section{Family—Parmeliaceae}

14. Bulbothrix isidiza (Nyl.) Hale, Phytologia 28: 480. 1974; D. D. Awasthi, Comp. Macrolich. India, Nepal \& Sri Lanka: 52. 2007. Parmelia isidiza Nyl. in Henriques, Bol. Soc. Brot. 3: 130. 1884.

Ecology and Distribution: On tree bark, rocks and soils, Karanjia, Jagatpur, Jamunadader, Mai-Ki-Bagia, Tikaritola, Sambhudhara, Dhunipani, Kapildhara, 850 - 1060 m.

Range of Distribution: India (Arunachal Pradesh, Karnataka, Kerala, Madhya Pradesh, Maharashtra, Manipur, Meghalaya, Nagaland, Sikkim, Tamil Nadu, Uttar Pradesh, Uttarakhand and West Bengal-foot hills), China, Japan, Nepal; widely distributed in Africa, South East Asia, Australia, Papua New Guinea; Central and South America.

Specimens examined: Karanjia, G. P. Sinha 1181 (BSA); A. N. Shukla 2908, 2915 (BSA); Jagatpur, A. N. Shukla 2940, 2924 (BSA); Jamunadader, A. N. Shukla 2556 (BSA); Mai-Ki-Bagia, G. P. Sinha 1145 (BSA); A. N. Shukla 1455 (BSA); Tikaritola, A. N. Shukla 2879 (BSA); Sambhudhara, A. N. Shukla 1496, 2508 (BSA); Dhuni pani, A. N. Shukla 2554, 2541 (BSA); Kapildhara, G. P. Sinha 1202 (BSA), det. A. N. Shukla.

15. Bulbothrix setschwanensis (Zahlbr.) Hale, Phytologia 28: 480. 1974; D. D. Awasthi, Comp. Macrolich. India, Nepal \& Sri Lanka: 54. 2007. Parmelia setchwa- 
nensis Zahlbr. in Handel-Mazzetti, Symb. Sin. 3: 182 \& 189. 1930.

Ecology and Distribution: On tree bark and rocks, Dhunipani, $900 \mathrm{~m}$.

Range of Distribution: India (Arunanchal Pradesh, Himachal Pradesh, Kerala, Madhya Pradesh, Manipur, Nagaland, Sikkim, Uttarakhand and West Bengal-hills), China and Nepal.

Specimen examined: Dhunipani, A. N. Shukla 2540 (BSA), det. A. N. Shukla.

16. Canoparmelia aptata (Kremp.) Elix \& Hale in Elix, Johnston \& Verdon, Mycotaxon 27: 278. 1986; D.D. Awasthi, Comp. Macrolich. India, Nepal \& Sri Lanka: 61. 2007. Parmelia aptata Kremp. In Nylander, Flora 52: 291. 1869.

Ecology and Distribution: On tree bark and rocks, Panchdhara, $950 \mathrm{~m}$.

Range of Distribution: India (Himachal Pradesh, Karnataka, Madhya Pradesh, Sikkim, Tamil Nadu, Uttarakhand and West Bengal-hills), Australia, Indonesia, Japan, Nepal, Philippines; Africa.

Specimen examined: Panchdhara, A. N. Shukla 2802 (BSA), det. A. N. Shukla.

17. Canoparmelia cinerascens (Lynge) Elix \& Hale in Elix, Johnston \& Verdon, Mycotaxon 27: 278. 1986; Divakar, Upreti \& Chatterjee, Geophtology 30(1-2): 4. 2002. Parmelia cinerascens Lynge, Ark. Bot. 30 B: 104. 1914.

Ecology and Distribution: On tree bark, Jagatpur, Karanjia, Tikaritola, Jamunadader, Sambhudhara, Panchdhara, 850 - $1060 \mathrm{~m}$.

Range of Distribution: India (Kerala, Madhya Pradesh and Tamil Nadu), Australia, Brazil, New Zealand, Paraguay.

Specimens examined: Jagatpur, A. N. Shukla 2930 (BSA); Karanjia, A. N. Shukla 2905, 2897 (BSA); Tikaritola, A. N. Shukla 2872, 2867, 2846 (BSA); Jamunadader, A. N. Shukla 2557, 2558, 2561 (BSA); Sambhudhara, A. N. Shukla 2505, 2510, 2514, 2495, 2484, 2506 (BSA); Panchdhara, A. N. Shukla 2820, 2800 (BSA), det. A. N. Shukla.

18. Canoparmelia crozalsiana (de Lesd.) Elix \& Hale, Mycotaxon 27: 278. 1986; D. D. Awasthi, Comp. Macrolich. India, Nepal \& Sri Lanka: 63. 2007. Parmelia crozalsiana de Lesd. ex Harm., Lich. France 4: 555. 1910.

Ecology and Distribution: On tree bark and rocks, Sambhudhara, $1002 \mathrm{~m}$.

Range of Distribution: India (Arunachal Pradesh, Kerala, Madhya Pradesh, Nagaland, Tamil Nadu and Uttarakhand), France, Italy, South Africa; North America and South America.

Specimen examined: Sambhudhara, A. N. Shukla 2486 (BSA), det. A. N. Shukla.

19. Canoparmelia eruptens (Kurok.) Elix \& Hale,
Mycotaxon 27: 278. 1986; D. D. Awasthi, Comp. Macrolich. India, Nepal \& Sri Lanka: 64. 2007. Parmelia eruptens Kurok. In Hale \& Kurokawa, Contr. US Natl. Herb. 36: 153.1964.

Ecology and Distribution: On tree bark, Amanala, Jaleswar, 900 - $910 \mathrm{~m}$.

Range of Distribution: India (Madhya Pradesh and Nagaland), Mozambique, New Zealand and South Africa.

Specimens examined: Amanala, A. N. Shukla 1537, 1535 (BSA); Jaleswar, A. N. Shukla 1536 (BSA), det. A. N. Shukla.

20. Canoparmelia texana (Tuck.) Elix \& Hale, Mycotaxon 27: 279. 1986; D. D. Awasthi, Comp. Macrolich. India, Nepal \& Sri Lanka: 65. 2007. Parmelia texana Tuck., Amer. J. Sci. Arts, ser. 2, 25: 424. 1858.

Ecology and Distribution: On tree bark and rocks, Karanjia, Jagatpur, Sonemuda, Dhunipani, Tikaritola, 850 - $1060 \mathrm{~m}$.

Range of Distribution: India (Chhattisgarh, Himachal Pradesh, Jammu \& Kashmir, Karnataka, Kerala, Madhya Pradesh, Maharashtra, Manipur, Meghalaya, Nagaland, Sikkim, Tamil Nadu and Uttarakhand); pantropical and subtemperate regions of the world especially in Africa, South and North America, Australia and New Zealand.

Specimens examined: Karanjia, G. P. Sinha 1180 (BSA); A. N. Shukla 2896, 2900 (BSA); Jagatpur, A. N. Shukla 2943 (BSA); Sonemuda, A. N. Shukla 2471, 2466, 2468 (BSA); Dhunipani, A. N. Shukla 2531 (BSA); Tikaritola, A. N. Shukla 2876, 2853 (BSA), det. A. N. Shukla.

21. Hypotrachyna awasthii Hale \& Patw., Bryologist 77: 637. 1974; D. D. Awasthi, Comp. Macrolich. India, Nepal \& Sri Lanka: 210. 2007.

Ecology and Distribution: On tree bark, Jamunadader, Tikaritola, Near Narmada temple, $1060 \mathrm{~m}$.

Range of Distribution: India (Karnataka, Kerala, Madhya Pradesh, Maharashtra, Manipur, Nagaland and Tamil Nadu). Endemic.

Specimens examined: Jamunadader, A. N. Shukla 2586, 2588 (BSA); Tikaritola, A. N. Shukla 2847 (BSA); Near Nermada temple, A. N. Shukla 2587 (BSA), det. A. N. Shukla.

22. Hypotrachyna crenata (Kurok.) Hale, Phytologia 28: 341. 1974; Patwardhan \& Prabhu, Curr. Sci. 46(6): 176. 1977. Parmelia crenata Kurok. in Hale \& Kurokawa, Contr. US Natl. Herb. 36: 168. 1964.

Ecology and Distribution: On tree bark and rocks, Karanjia, $850 \mathrm{~m}$.

Range of Distribution: India (Himachal Pradesh, Karnataka, Kerala, Madhya Pradesh, Maharashtra, Meghalaya, Sikkim, Tamil Nadu, Uttarakhand and West Bengalhills), Japan, Nepal, Taiwan and Thailand.

Specimen examined: Karanjia, A. N. Shukla 2899 (BSA), det. A. N. Shukla.

23. Myelochroa aurulenta (Tuck.) Elix \& Hale, My- 
cotaxon 29: 240. 1987; D. D. Awasthi, Comp. Macrolich. India, Nepal \& Sri Lanka: 290. 2007. Parmelia aurulenta Tuck., Amer. J. Sci. Arts, ser. 2, 25: 424. 1858.

Ecology and Distribution: On tree bark and rocks, Near Narmada temple, $1060 \mathrm{~m}$.

Range of Distribution: India (Arunachal Pradesh, Himachal Pradesh, Kerala, Madhya Pradesh, Manipur, Nagaland, Sikkim, Tamil Nadu, Uttarakhand and West Bengal-hills); Australia; Eastern Africa, North America, South America, S. E. Asia.

Specimen examined: Near Nermada temple, A.N. Shukla 1478 (BSA), det. A. N. Shukla.

24. Parmelinopsis cryptochlora (Vain.) Elix \& Hale, Mycotaxon 29: 242. 1987; D. D. Awasthi, Comp. Macrolich. India, Nepal \& Sri Lanka: 325. 2007. Parmelia cryptochlora Vain., J. Bot. Brit. Foreign 34: 34. 1896.

Ecology and Distribution: On tree bark, Helipad forest area, $1070 \mathrm{~m}$.

Range of Distribution: India (Madhya Pradesh and Tamil Nadu), Australia, Carribean Islands and Papua New Guinea.

Specimen examined: Helipad forest area, G. P. Sinha 1206 (BSA), det. A. N. Shukla.

25. Parmotrema praesorediosum (Nyl.) Hale, Phytologia 28: 338. 1974; D. D. Awasthi, Comp. Macrolich. India, Nepal \& Sri Lanka: 347. 2007. Parmelia praesorediosa Nyl., Sert. Lich. Trop.: 18. 1891.

Ecology and Distribution: On tree bark and rocks, Jagatpur, Sonemuda, Tikaritola, Mai-Ki-Bagia, Sambhudhara, Jamunadader, Antaria, Lamni, Ammanala, 910 $1060 \mathrm{~m}$.

Range of Distribution: India (Andhra Pradesh, Assam, Himachal Pradesh, Jammu \& Kashmir, Karnataka, Kerala, Madhya Pradesh, Maharashtra, Manipur, Nagalnd, Orissa, Rajasthan, Tamil Nadu, Uttarakhand, Uttar Pradesh and West Bengal); tropical regions of the world.

Specimens examined: Jagatpur, A. N. Shukla 2934, 2945 (BSA); Sonemuda, A. N. Shukla 1472 (BSA); Tikaritola, A. N. Shukla 2833, 2837, 2864 (BSA); Mai-KiBagia, A. N. Shukla 1450, 1457 (BSA); Sambhudhara, A. N. Shukla 2503 (BSA); Jamunadader, A. N. Shukla 2604 (BSA); Antaria, A. N. Shukla 2622, 2624, 2641, 2653 (BSA); Lamni, A. N. Shukla 2700, 2716, 2722, 2731 (BSA); Ammanala, A. N. Shukla 1534 (BSA), det. A. $N$. Shukla.

26. Parmotrema rampoddense (Nyl.) Hale, Phytologia 28: 338. 1974; D. D. Awasthi, Comp. Macrolich. India, Nepal \& Sri Lanka: 349. 2007. Parmelia rampoddensis Nyl., Acta Soc. Sci. Fenn. 26 (10): 7. 1900.

Ecology and Distribution: On tree bark, Panchdhara, $950 \mathrm{~m}$.

Range of Distribution: India (Karnataka, Kerala, Madhya Pradesh, Maharashtra, Manipur, Nagaland, Sikkim, Tamil Nadu, Uttarakhand and West Bengal), Nepal, Sri
Lanka; tropical regions of the world.

Specimen examined: Panchdhara, A. N. Shukla 2805 (BSA), det. A. N. Shukla.

27. Parmotrema saccatilobum (Taylor) Hale, Phytologia 28: 339, 1974; D. D. Awasthi, Comp. Macrolich. India, Nepal \& Sri Lanka: 351. 2007. Parmelia saccatiloba Taylor in Hooker, London J. Bot. 6: 174. 1847.

Ecology and Distribution: On tree bark, Jamunadader, Sambhudhara, Mai-Ki-Bagia, 960 - 1060 m.

Range of Distribution: India (Andaman \& Nicobar Islands, Assam, Goa, Kerala, Madhya Pradesh, Maharashtra, Nagaland, Sikkim, Uttarakhand and West Bengalplains), Australia, China, Fiji, Singapore, Taiwan; Pacific Islands.

Specimens examined: Jamunadader, A. N. Shukla 2584 (BSA); Sambhudhara, A. N. Shukla 2481 (BSA); Mai-KiBagia, A. N. Shukla 1464 (BSA), det. A. N. Shukla.

28. Parmotrema tinctorum (Despr. Ex Nyl.) Hale, Phytologia 28: 33. 1974; D. D. Awasthi, Comp. Macrolich. India, Nepal \& Sri Lanka: 354. 2007. Parmelia tinctoria Despr. ex Nyl., Flora 55: 547. 1872.

Ecology and Distribution: On tree bark and soils, Jagatpur, Karanjia, Panchdhara, Mai-Ki-Bagia, Kabirchabutara, Tikaritola, 910 - $1060 \mathrm{~m}$.

Range of Distribution: India (Andhra Pradesh, Arunachal Pradesh, Assam, Chhattisgarh, Himachal Pradesh, Jammu \& Kashmir, Jharkhand, Karnataka, Kerala, Madhya Pradesh, Maharashtra, Manipur, Meghalaya, Nagaland, Orissa, Rajasthan, Sikkim, Tamil Nadu, Uttarakhand and West Bengal); widely distributed in Australia, China, Indonesia, New Zealand; Africa, North, Central and South America.

Specimens examined: Jagatpur, A. N. Shukla 2942, 2931 (BSA); Karanjia, A. N. Shukla 2917 (BSA); Panchdhara, A. N. Shukla 2795, 2801 (BSA); Mai-Ki-Bagia, G. P. Sinha 1147 (BSA); Kabirchabutra, G. P. Sinha 1173 (BSA); Tikaritola, A. N. Shukla 2834, 2858 (BSA), det. A. N. Shukla.

29. Phyllopsora corallina (Eschw.) Müll.Arg., Bot. Jaarb. 20: 264. 1894; D. D. Awasthi, Beih. Nova Hedwigia 17: 98. 1965. Lecidea ontanae Eschw. in Martius, Fl. Bras. 1: 256. 1833.

Ecology and Distribution: On tree bark, Near Nermada temple, $1060 \mathrm{~m}$.

Range of Distribution: India (Arunachal Pradesh, Goa, Himachal Pradesh, Karnataka, Madhya Pradesh, Maharashtra, Sikkim, Tamil Nadu and Uttarakhand); Central and Southern America.

Specimen examined: Near Nermada temple, A.N. Shukla 1487 (BSA), det. A. N. Shukla.

\section{Family-Pertusariaceae}

30. Ochrolechia androgyna (Hoffm.) Arnold, Flora 68: 236. 1855; D. D. Awasthi \& Tewari, Kavaka 15(1-2): 24. 1987. Lichen androgynus Hoffm., Enum. Lich.: 56. 
1784.

Ecology and Distribution: On tree bark, Karanjia, $850 \mathrm{~m}$.

Range of Distribution: India (Karnataka and Madhya Pradesh); boreal to arctic circumpolar with a notable disjunct south western population especially along the Sierra Nevada mountains.

Specimen examined: Karanjia, G. P. Sinha 1186 (BSA), det. A. N. Shukla.

31. Pertusaria colorata D. D. Awasthi \& P. Srivastava, Bryologist 96(2): 210. 1993.

Ecology and Distribution: On tree bark, Jamunadader, $1060 \mathrm{~m}$.

Range of Distribution: India (Karnataka, Madhya Pradesh and Tamil Nadu). Endemic.

Specimens examined: Jamunadader, A. N. Shukla 2595, 2570 (BSA), det. A. N. Shukla.

32. Pertusaria concinna Erichsen, Repert. Spec. Nov. Regni Veg. 41: 79. 1936; D. D. Awasthi, Beih. Nova Hedwigia 17: 92. 1965 \& Biblioth. Lichenol. 40: 212. 1991.

Ecology and Distribution: On tree bark, Karanjia, Panchdhara, Dhunipani, Jamunadader, Sonemuda, Sambhudhara, 850 - $1060 \mathrm{~m}$.

Range of Distribution: India (Chhattisgarh, Himachal Pradesh, Karnataka, Madhya Pradesh and Tamil Nadu). Endemic.

Specimens examined: Karanjia, A. N. Shukla 1187, 2922, 2891 (BSA); Panchdhara, A. N. Shukla 2811, 2810 (BSA); Dhunipani, A. N. Shukla 2522, 2525 (BSA); Jamunadader, A. N. Shukla 2593, 2610 (BSA); Sonemuda, A. N. Shukla 2460 (BSA); Sambhudhara, A. N. Shukla 2516 (BSA), det. A. N. Shukla.

33. Pertusaria depressa (Fée.) Mont \& Bosch in Miquel, Pl. Jungh. 4: 482. 1857; D. D. Awasthi, Beih. Nova Hedwigia 17: 92. 1965. Porina depressa Fée, Essai Crypt. Ecorc.: 80, t. 20, f. 2, 1824.

Ecology and Distribution: On tree bark, Sonemuda, $950 \mathrm{~m}$.

Range of Distribution: India (Madhya Pradesh and Tamil Nadu); tropical regions of the world.

Specimen examined: Sonemuda, A. N. Shukla 2473 (BSA), det. A. N. Shukla.

34. Pertusaria granulata (Ach.) Müll.Arg., Flora 68: 354. 1884; D. D. Awasthi, Biblioth. Lichenol. 40: 220. 1991. Porina granulata Ach., Syn. Lich.: 112. 1814.

Ecology and Distribution: On tree bark, Panchdhara, Dhunipani, Jamunadader, Sonemuda, 950 - 1060 m.

Range of Distribution: India (Karnataka, Kerala, Madhya Pradesh and Tamil Nadu), Sri Lanka.

Specimens examined: Panchdhara, A. N. Shukla 2789, 2817, 2825 (BSA); Dhunipani, A. N. Shukla 2545 (BSA); Jamunadader, A. N. Shukla 2596, 2566 (BSA); Sonemuda, A. N. Shukla 2444, 2447 (BSA), det. A. N. Shukla.

35. Pertusaria leucostoma (Bernh.) A.Massal., Ric.
Auton. Lich. Crost.: 188, 387. 1852. Sphaeria leucostoma Bernh. In Roemer, Arch. Bot. (Leipzig) 2: 11, Table 1, Figure 1, 2. 1799. Pertusaria acuta Müll.Arg., Flora 67: 353. 1884; D. D. Awasthi, Beih. Nova Hedwigia 17: 92. 1965.

Ecology and Distribution: On tree bark, Karangia, $850 \mathrm{~m}$.

Range of Distribution: India (Himachal Pradesh, Madhya Pradesh, Manipur, Orissa, Rajasthan and Tamil Nadu), Australia, Brazil, Nepal and Sri Lanka.

Specimen examined: Karangia, A. N. Shukla 2918 (BSA), det. A. N. Shukla.

36. Pertusaria leucosora Nyl., Flora 60: 223. 1877; D. D. Awasthi, Biblioth. Lichenol. 40: 209. 1991.

Ecology and Distribution: On rocks, Sambhudhara, $1002 \mathrm{~m}$.

Range of Distribution: India (N. W. Himalaya, Karnataka, Kerala, Madhya Pradesh and Tamil Nadu); Europe.

Specimen examined: Sambhudhara, A. N. Shukla 1500 (BSA), det. A. N. Shukla.

37. Pertusaria leucosorodes Nyl., Lich. Jap.: 56. 1890;

D. D. Awasthi, Beih. Nova Hedwigia 17: 93. 1965.

Ecology and Distribution: On tree bark, Sambhudhara, $1002 \mathrm{~m}$.

Range of Distribution: India (E. and N. W. Himalaya, Madhya Pradesh, Karnataka, Kerala, Nagaland and Tamil Nadu), Nepal and Sri Lanka.

Specimens examined: Sambhudhara, A. N. Shukla 2488, 2494 (BSA), det. A. N. Shukla.

38. Pertusaria melastomella Nyl., Acta Soc. Sci. Fenn. 26(10): 16. 1900; D. D. Awasthi, Biblioth. Lichenol. 44: 222. 1991.

Ecology and Distribution: On tree bark, Sambhudhara, Mai-Ki-Bagia, Jamunadader, Amarkantak helipad area, 960 - $1060 \mathrm{~m}$.

Range of Distribution: India (Himachal Pradesh, Madhya Pradesh and Tamil Nadu) and Sri Lanka.

Specimens examined: Sambhudhara, A. N. Shukla 1501 (BSA); Mai-Ki-Bagia, G. P. Sinha 1150 (BSA); Jamunadader, A. N. Shukla 2611, 2569 (BSA); Amarkantak helipad area, G. P. Sinha 1207 (BSA), det. A. N. Shukla.

39. Pertusaria neilgherrensis (Müll.Arg.) D. D. Awasthi \& P. Srivastava in D. D. Awasthi, Biblioth. Lichenol. 40: 3, 213. 1991. Pertusaria leioplacoides var. Neilgherrensis Müll.Arg., Flora 67: 301. 1884.

Ecology and Distribution: On tree bark, Tikaritola, $1060 \mathrm{~m}$.

Range of Distribution: India (Madhya Pradesh and Tamil Nadu). Endemic.

Specimen examined: Tikaritola, A. N. Shukla 2883 (BSA), det. A. N. Shukla.

40. Pertusaria pertusa (L.) Tuck., Enum. N. America. Lich.: 56. 1845; D. D. Awasthi, Biblioth. Lichenol. 40: 214. 1991. Lichen pertusus L., Mantissa Pl.: 131. 1767. Sphaeria pertusa Weigel, Observ. Bot.:46. 1772. 
Ecology and Distribution: On tree bark, Dhunipani, Sambhudhara, 900 - 1002 m.

Range of Distribution: India (Himachal Pradesh, Kerala, Madhya Pradesh, Maharashtra and Rajasthan), Nepal, Sri Lanka; tropical regions.

Specimens examined: Dhunipani, A. N. Shukla 2555 (BSA); Sambhudhara, A. N. Shukla 2502 (BSA), det. A. N. Shukla.

41. Pertusaria punctata. Nyl., Bull. Soc. Linn. Normandie, ser. 2, 2: 71. 1868; D. D. Awasthi, Beih. Nova Hedwigia 17: 93. 1965.

Ecology and Distribution: On tree bark, Panchdhara, $950 \mathrm{~m}$.

Range of Distribution: India (Himachal Pradesh, Karnataka, Madhya Pradesh and Tamil Nadu). Endemic.

Specimens examined: Panchdhara, A. N. Shukla 2830, 2826, 2819 (BSA), det. A. N. Shukla.

42. Pertusaria quassiae (Fée) Nyl., Ann. Sci. Nat., Bot., ser. 4, 15: 45. 1861; D. D. Awasthi, Biblioth. Lichenol. 40: 212. 1991. Porina quassiae Fée, Essai Crypt. Ecorc.: 81. 1824.

Ecology and Distribution: On tree bark, Sonemuda, Tikaritola, 950 - $1060 \mathrm{~m}$.

Range of Distribution: India (Arunachal Pradesh, Andaman \& Nicobar Islands, Andhra Pradesh, Himachal Pradesh, Jammu \& Kashmir, Karnataka, Madhya Pradesh, Maharashtra and Nagaland), Nepal, Sri Lanka; tropical regions of the world.

Specimens examined: Sonemuda, A. N. Shukla 2461 (BSA); Tikaritola, A. N. Shukla 2855, 2844, 2849, 2852 (BSA), det. A. N. Shukla.

43. Pertusaria rigida Müll.Arg., J. Linn. Soc., Bot. 29: 221. 1893; D. D. Awasthi, Beih. Nova Hedwigia 17: 93. 1965.

Ecology and Distribution: On tree bark, Karangia, Dhunipani, Sonemuda, 850 - 950 m.

Range of Distribution: India (Madhya Pradesh and Manipur) and Australia.

Specimens examined: Karangia, A. N. Shukla 2890 (BSA); Dhunipani, A. N. Shukla 2535, 2523 (BSA); Sonemuda, A. N. Shukla 2770 (BSA), det. A. N. Shukla.

44. Pertusaria rimosa D. D. Awasthi \& P. Srivastava, Bryologist 96(2): 214. 1993.

Ecology and Distribution: On tree bark, Jamunadader, Mai-Ki-Bagia, Karanjia, 850 - 1060 m.

Range of Distribution: India (Madhya Pradesh and Tamil Nadu). Endemic.

Specimens examined: Jamunadader, A. N. Shukla 2605 (BSA); Mai-Ki-Bagia, A. N. Shukla 1460 (BSA); Karanjia, G. P. Sinha 1182 (BSA), det. A. N. Shukla.

45. Pertusaria tetrathalmia (Fée) Nyl., Acta Soc. Sci. Fenn. 7: 448. 1863. Trypethelium tetrathalmium Fée, Essai Crypt. Ecorc.: 69. 1824. Pertusaria tuberculifera Nyl., Acta. Soc. Sci. Fenn. 7: 448. 1863; D. D. Awasthi, Bib- lioth. Lichenol. 40: 220. 1991.

Ecology and Distribution: On tree bark, Panchdhara, Karanjia, Sonemuda, 850 - 950 m.

Range of Distribution: India (Karnataka, Kerala, Madhya Pradesh and Tamil Nadu) and Sri Lanka.

Specimens examined: Dhunipani, A. N. Shukla 2524 (BSA); Panchdhara, A. N. Shukla 2829 (BSA); Karanjia, A. N. Shukla 2892 (BSA); Sonemuda, A. N. Shukla 2469, 2445 (BSA); G. P. Sinha 1174 (BSA), det. A. N. Shukla.

\section{Family-Physciaceae}

46. Amandinea diorista (Nyl.) Marbach, Biblioth. Lichenol. 74: 58. 2000. Lecidea diorista Nyl., Flora 52: 71. 1869. Buellia diorista (Nyl.) Zahlbr., Cat. Lich. Univ. 7: 349. 1931; D. D. Awasthi, Beih. Nova Hedwigia 17: 31. 1965.

Ecology and Distribution: On tree bark, Forest medicinal garden, $1060 \mathrm{~m}$.

Range of Distribution: India (Madhya Pradesh, Manipur and West Bengal), Belgium, Guatemala and Pacific region.

Specimen examined: Forest medicinal garden, $A . N$. Shukla 1510 (BSA), det. A. N. Shukla.

47. Amandinea montana (H.Magn.) Marbach, Biblioth. Lichenol. 74: 93. 2000. Buellia ontana H. Magn., Bot. Not. 2: 199. 1954; D. D. Awasthi, Beih. Nova Hedwigia 17: 32. 1965.

Ecology and Distribution: On tree bark, Jagatpur, 910 m.

Range of Distribution: India (Himachal Pradesh, Jammu \& Kashmir, Madhya Pradesh, Manipur and Uttarakhand) and Kenya.

Specimens examined: Jagatpur, A. N. Shukla 2941, 2947 (BSA), det. A. N. Shukla.

48. Amandinea punctata (Hoffm.) Coppins \& Scheid., Lichenologist 25(4): 343. 1993. Verrucaria punctata Hoffm., Deutschl. Fl.: 192. 1796. Buellia punctata (Hoffm.) A.Massal., Ric. Auton. Lich. Crost.: 81 fig. 165, 1852; S. Singh \& D. D. Awasthi, Biol. Mem. 6(2): 183. 1981.

Ecology and Distribution: On tree bark, Jagatpur, $910 \mathrm{~m}$.

Range of Distribution: India (Himachal Pradesh, Jammu \& Kashmir, Madhya Pradesh and Manipur), Australia and Brazil.

Specimen examined: Jagatpur, A. N. Shukla 2938 (BSA), det. A. N. Shukla.

49. Buellia alboatra (Hoffm.) Th.Fr., Gen. Heterolich. Eur.: 91. 1861; Kr. P. Singh, Geophytology 10(1-2): 272. 1980. Lichen alboater Hoffm., Enum. Lich. Eur.: 30. 1784.

Ecology and Distribution: On tree bark and rocks, Amarkantak helipad area, $1060 \mathrm{~m}$.

Range of Distribution: India (Arunachal Pradesh, Jammu \& Kashmir, Madhya Pradesh, Manipur and Uttarakhand); temperate regions.

Specimen examined: Amarkantak helipad area, G. P. Sinha 1204 (BSA), det. A. N. Shukla. 
50. Buellia betulinoides R. Schub. \& Klement, Nova Hedwigia 11: 66. 1966; S. Singh \& D. D. Awasthi, Biol. Mem. 6(2):174. 1981.

Ecology and Distribution: On tree bark, Forest medicinal garden, $1060 \mathrm{~m}$.

Range of Distribution: India (Jammu \& Kashmir, Madhya Pradesh and Tamil Nadu). Endemic.

Specimen examined: Forest medicinal garden, A. N. Shukla 1507 (BSA), det. A. N. Shukla.

51. Buellia quartziana S. R. Singh \& D. D. Awasthi, Biol. Mem. 6(2): 189. 1981.

Ecology and Distribution: On tree bark, Sambhudhara, $1002 \mathrm{~m}$.

Range of Distribution: India (Madhya Pradesh). Endemic.

Specimen examined: Sambhudhara, G. P. Sinha 2496 (BSA), det. A. N. Shukla.

52. Cratiria obscurior (Stirt.) Marbach \& Kalb in Marbach, Biblioth. Lichenol. 74: 186. 2000. Pyxine obscurior Stirt., Trans. Roy. Soc. Victoria 17: 70. 1881. Buellia inornata (Stirt.) Zahlbr., Cat. Lich. Univ. 7: 369. 1931; D. D. Awasthi, Beih. Nova Hedwigia 17: 31. 1965.

Ecology and Distribution: On tree bark, Sambhudhara, $1002 \mathrm{~m}$.

Range of Distribution: India (Andhra Pradesh, Assam, Bihar, Kerala, Madhya Pradesh, Tamil Nadu, Uttarakhand and West Bengal), Nepal, Philippines, Thailand; Africa and South America.

Specimen examined: Sambhudhara, A. N. Shukla 2496 (BSA), det. A. N. Shukla.

53. Dirinaria applanata (Fée) D. D. Awasthi in D.D. Awasthi \& Agarwal, J. Indian Bot. Soc. 49: 135. 1970. Parmelia applanta Fée, Essai Crypt. Ecorc.: 126. 1824, tab. 32, fig. 2 \& Suppl.: 123, tab. 42, fig. 18. 1837.

Ecology and Distribution: On tree bark and rocks, Karangia, Jamunadader, Lamni, Jalda, Antaria, 440 - 1060 m.

Range of Distribution: India (Andaman \& Nicobar Islands, Chhattisgarh, Karnataka, Madhya Pradesh, Maharashtra, Nagaland, Sikkim, Tamil Nadu, Uttar Pradesh, Uttarakhand and West Bengal-plains), Sri Lanka; widely distributed in the tropical and subtropical regions of the world.

Specimens examined: Karangia, A. N. Shukla 2895 (BSA); Jamunadader, A. N. Shukla 2770 (BSA); Lamni, A. N. Shukla 2694, 2728 (BSA); Jalda, A. N. Shukla 2740, 2773, 2765 (BSA); Antaria, A. N. Shukla 2677, 2657 (BSA), det. A. N. Shukla.

54. Dirinaria consimilis (Stirt.) D. D. Awasthi in D.D. Awasthi \& Agarwal, J. Indian Bot. Soc. 49: 135. 1970. Physcia consimilis Stirt., Proc. Roy. Soc. Glasgow 11: 310. 1879.

Ecology and Distribution: On tree bark and rocks, Karangia, Jagatpur, Lamni, Panchdhara, Dhunipani, Samhudhara, Jamunadader, Sonemuda, 660 - 1060 m.
Range of Distribution: India (Arunachal Pradesh, Chhattisgarh, Himachal Pradesh, Kerala, Madhya Pradesh, Manipur, Nagaland, Sikkim, Tamil Nadu, Uttar Pradesh and West Bengal), Nepal; widely distributed in the tropical region of Africa and Asia.

Specimens examined: Karangia, G. P. Sinha 1185 (BSA); Jagatpur, A. N. Shukla 2932 (BSA); Lamni, A. N. Shukla 2703 (BSA); Panchdhara, A. N. Shukla 2813, 2831, 2783 (BSA); Dhunipani, A. N. Shukla 2528, 2549 (BSA); Samhudhara, A. N. Shukla 2479 (BSA); Jamunadader, A. N. Shukla 2579 (BSA); Sonemuda, A. N. Shukla 2452 (BSA), det. A. N. Shukla.

55. Hafellia disciformis (Fr.) Marbach \& H. Mayrhofer in Marbach, Biblioth. Lichenol. 74: 264. 2000. Lecidea parasema var. disciformis Fr., Nov. Sched. Critic: 9. 1826. Buellia disciformis (Fr.) Mudd, Man. Brit. Lich.: 216. 1861; D. D. Awasthi, Beih. Nova Hedwigia 17: 31. 1965.

Ecology and Distribution: On tree bark, Jagatpur, Karanjia, 850 - $910 \mathrm{~m}$.

Range of Distribution: India (Arunachal Pradesh, Himachal Pradesh, Madhya Pradesh, Manipur, Tamil Nadu and West Bengal-hills). Cosmopolitan.

Specimens examined: Jagatpur, A. N. Shukla 2946, 2929 (BSA); Karanjia, A. N. Shukla 2907 (BSA), det. A. N. Shukla.

56. Heterodermia albidiflava (Kurok.) D. D.Awasthi, Geophytology 3: 113. 1973. Anaptychia albidiflava Kurok., Beih. Nova Hedwigia 6: 42. 1962.

Ecology and Distribution: On tree bark, Panchdhara, $950 \mathrm{~m}$.

Range of Distribution: India (Himachal Pradesh, Madhya Pradesh, Sikkim and West Bengal-hills). Endemic.

Specimen examined: Panchdhara, G. P. Sinha 2828 (BSA), det. A. N. Shukla.

57. Heterodermia angustiloba (Müll.Arg.) D. D. Awasthi, Geophytology 3: 113. 1973. Anaptychia speciosa var. Angustiloba Müll.Arg., Flora 66: 78. 1883.

Ecology and Distribution: On tree bark, Panchdhara, Sambhudhara, Mai-Ki-Bagia, 950 - 1002 m.

Range of Distribution: India (Arunachal Pradesh, Himachal Pradesh, Karnataka, Madhya Pradesh, Maharashtra, Sikkim and Uttarakhand), Australia, China, Japan, Nepal and Taiwan.

Specimens examined: Panchdhara, A. N. Shukla 2832 (BSA); Sambhudhara, A. N. Shukla 1505 (BSA); Mai-KiBagia, G. P. Sinha 1152 (BSA), det. A. N. Shukla.

58. Heterodermia boryi (Fée) Kr. P. Singh \& S. R. Singh, Geophytology 6: 33. 1976. Borrera boryi Fée, Essai Crypt. Ecorc.: 92, Table 2, Figure 23. 1824.

Ecology and Distribution: On tree bark and soils, Sambhudhara, $1002 \mathrm{~m}$.

Range of Distribution: India (Jammu \& Kashmir, Kerala, Madhya Pradesh, Maharashtra, Nagaland, Sikkim, 
Tamil Nadu and Uttarakhand); widely distributed in tropical and temperate regions of the world.

Specimen examined: Sambhudhara, A. N. Shukla 2500 (BSA), det. A. N. Shukla.

59. Heterodermia dactyliza (Nyl.) Swinscow \& Krog, Lichenologist 8: 117. 1976; D. D. Awasthi, Comp. Macrolich. India, Nepal \& Sri Lanka: 181. 2007. Physcia speciosa var. Dactyliza Nyl., Syn. Lich. 1: 417. 1860.

Ecology and Distribution: On tree bark and rocks, Karanjia, $850 \mathrm{~m}$.

Range of Distribution: India (Kerala, Madhya Pradesh, Sikkim and West Bengal-hills), Nepal; South America.

Specimen examined: Karanjia, A. N. Shukla 2904, 2923 (BSA), det. A. N. Shukla.

60. Heterodermia diademata (Taylor) D. D. Awasthi, Geophytology 3: 113. 1973. Parmelia diademata Taylor, London J. Bot. 6: 165. 1847.

Ecology and Distribution: On tree bark and rocks, Karangia, Sambhudhara, Jamunadader, Sonemuda, Near Narmada temple, 850 - $1060 \mathrm{~m}$.

Range of Distribution: India (Arunachal Pradesh, Assam, Himachal Pradesh, Jammu \& Kashmir, Karnataka, Kerala, Madhya Pradesh, Maharashtra, Manipur, Meghalaya, Nagaland, Rajasthan, Sikkim, Tamil Nadu,Uttarakhand and West Bengal-hills); widely distributed in Asia (China, Japan, Nepal, Sri Lanka, Taiwan), Africa, Central and South America.

Specimens examined: Karangia, A. N. Shukla 2919, 2906, 2903 (BSA); Sambhudhara, A. N. Shukla 2504 (BSA); Jamunadader, A. N. Shukla 2574 (BSA); Sonemuda, A. N. Shukla 1475 (BSA); Near Nermada temple, A. N. Shukla 1482, 1513 (BSA), det. A. N. Shukla.

61. Heterodermia dissecta (Kurok.) D. D. Awasthi, Geophytology 3: 113. 1973. Anaptychia dissecta Kurok., J. Jap. Bot. 34: 182. 1959.

Ecology and Distribution: On tree bark and soils, Sambhudhara, Kapildhara, Karanjia, Jagatpur, 850 - 1002 m.

Range of Distribution: India (Himachal Pradesh, Karnataka, Kerala, Madhya Pradesh, Manipur, Nagaland, Sikkim, Tamil Nadu, Uttarakhand and West Bengal-hills), China, Japan, Nepal, Taiwan and Central America.

Specimens examined: Sambhudhara, A. N. Shukla 2499 (BSA); Kapildhara, G. P. Sinha 1199 (BSA); Karanjia, A. N. Shukla 2911 (BSA); Jagatpur, A. N. Shukla 2948 (BSA), det. A. N. Shukla.

62. Heterodermia hypocaesia (Yasuda) D. D. Awasthi, Geophytology 3: 113. 1973. Anaptychia hypocaesia Yasuda in Räsänen, J. Jap. Bot. 16: 139. 1940.

Ecology and Distribution: On tree bark, rocks and soils, Sambhudhara, Amarkantak helipad area, 1002 - 1060 m.

Range of Distribution: India (Himachal Pradesh, Kerala, Madhya Pradesh, Manipur, Nagaland, Sikkim, Tamil Nadu, Uttarakhand and West Bengal-hills), Australia, China, Hawaii, Japan, Indonesia, Philippines; Africa.
Specimens examined: Sambhudhara, A. N. Shukla 1504 (BSA); Amarkantak helipad area, G. P. Sinha 1203 (BSA), det. A. N. Shukla.

63. Heterodermia obscurata (Nyl.) Trevis., Nouvo Giorn Bot. Ital 1: 114. 1869; D. D. Awasthi, Comp. Macrolich. India, Nepal \& Sri Lanka: 190. 2007. Physcia obscurata Nyl., Ann. Sci. Nat., Bot., ser. 4, 19; 310. 1863.

Ecology and Distribution: On tree bark, rocks and soils, Panchdhara, Sonemuda, Mai-Ki-Bagia, Jamunadader, Jagatpur, 910 - $1060 \mathrm{~m}$.

Range of Distribution: India (Arunachal Pradesh, Himachal Pradesh, Jammu \& Kashmir, Kerala, Madhya Pradesh, Maharashtra, Manipur, Nagaland, Sikkim, Tamil Nadu, Uttarakhand and West Bengal-hills), China, Hawaii, Japan, Nepal, New Zealand, Philippines, Thailand; Africa, North, Central and South America.

Specimens examined: Panchdhara, A. N. Shukla 2791, 2781 (BSA); Sonemuda, A. N. Shukla 1474 (BSA); MaiKi-Bagia, A. N. Shukla 1454 (BSA); Jamunadader, A. $N$. Shukla 2608 (BSA); Jagatpur, A. N. Shukla 2944 (BSA), det. A. N. Shukla.

64. Heterodermia pseudospeciosa (Kurok.) W.L. Culb., Bryologist 69: 484. 1966; D. D. Awasthi, Comp. Macrolich. India, Nepal \& Sri Lanka: 193. 2007. Anoptychia pseudospeciosa Kurok., J. Jap.Bot. 34: 176. 1959.

Ecology and Distribution: On tree bark, rocks and soils, Amarkantak helipad area, $1060 \mathrm{~m}$.

Range of Distribution: India (Himachal Pradesh, Kerala, Madhya Pradesh, Maharashtra, Manipur, Nagaland, Sikkim, Tamil Nadu, Uttarakhand and West Bengal-hills), China, Hawaii Islands, Japan, Nepal, Taiwan; Africa, Central and South America.

Specimen examined: Amarkantak helipad area, G. P. Sinha 2845 (BSA), det. A. N. Shukla.

65. Heterodermia rubescens (Räsänen) D. D. Awasthi, Geophytology 3: 114. 1973. Anaptychia hypoleuca f. Rubescens Räsänen, Suom. Elain-ja Kasvit. Seuran Van. Tiedon. Pöytäkirjat 5: 27. 1950.

Ecology and Distribution: On tree bark and rocks, Panchdhara, $950 \mathrm{~m}$.

Range of Distribution: India (Arunachal Pradesh, Himachal Pradesh, Madhya Pradesh, Manipur, Meghalaya, Nagaland, Rajashtan, Sikkim, Uttarakhand and West Bengal-hills), China, Nepal and Taiwan.

Specimen examined: Panchdhara, A. N. Shukla 2790 (BSA), det. A. N. Shukla.

66. Heterodermia speciosa (Wulfen) Trevis., Atti Soc. Ital. Sci. Nat. 11: 614. 1868; D. D. Awasthi, Comp. Macrolich. India, Nepal \& Sri Lanka: 195. 2007. Lichen spe- ciosa Wulfen in Jacquin, Coll. Bot. 3: 119. 1789.

Ecology and Distribution: On tree bark and rocks, Sambhudhara, $1002 \mathrm{~m}$.

Range of Distribution: India (Arunachal Pradesh, Himachal Pradesh, Jammu \& Kashmir, Madhya Pradesh, 
Maharashtra, Manipur, Nagaland, Sikkim, Tamil Nadu, Uttarakhand and West Bengal-hills); tropical regions of the world.

Specimen examined: Sambhudhara, A. N. Shukla 1494 (BSA), det. A. N. Shukla.

67. Heterodermia tremulans (Müll.Arg.) W.L.Culb., Bryologist 69: 485. 1966; D. D. Awasthi, Comp. Macrolich. India, Nepal \& Sri Lanka: 196. 2007. Physcia hypoleuca var. Tremulans Müll.Arg., Flora 63: 277. 1880.

Ecology and Distribution: On tree bark, rocks and soils, Amarkantak helipad area, $1060 \mathrm{~m}$.

Range of Distribution: India (Karnataka, Madhya Pradesh, Nagaland, Rajasthan, Sikkim, Tamil Nadu, Uttarakhand and West Bengal-hills), China, Hawaii, Japan; North, Central and South America.

Specimen examined: Amarkantak helipad area, G. P. Sinha 1204 (BSA), det. A. N. Shukla.

68. Phaeophyscia endococcina (Körb.) Moberg, Symb. Bot. Upsal 22(1): 35. 1977; D. D. Awasthi, Comp. Macrolich. India, Nepal \& Sri Lanka: 372. 2007. Parmelia endococcina Körb., Parerga Lichenol: 36. 1859.

Ecology and Distribution: On tree bark and rocks, Near Narmada temple, $1060 \mathrm{~m}$.

Range of Distribution: India (Arunachal Pradesh, Himachal Pradesh, Jammu \& Kashmir, Madhya Pradesh, Sikkim and Uttarakhand); cosmopolitan in northern temperate parts of the world.

Specimen examined: Near Narmada temple, A.N. Shukla 1486 (BSA).

69. Phaeophyscia orbicularis (Neck.) Moberg, Symb. Bot. Upsal. 22(1): 44. 1977; D. D. Awasthi, Comp. Macrolich. India, Nepal \& Sri Lanka: 375. 2007. Lichen orbicularis Neck., Delic. Gallo-Belg.: 509. 1768.

Ecology and Distribution: On tree bark and rocks, Helipad area, $1060 \mathrm{~m}$.

Range of Distribution: India (Himachal Pradesh, Jammu \& Kashmir, Madhya Pradesh, Manipur, Nagaland, Sikkim, Tamil Nadu, Uttar Pradesh and Uttarakhand), New Zealand; Europe, North America.

Specimen examined: Helipad area, G. P. Sinha 1211 (BSA), det. A. N. Shukla.

70. Physcia dimidiata (Arn.) Nyl., Flora 64: 537. 1881; G. P. Sinha \& Kr. P. Singh, Macrolich. Sikkim: 195. 2005. Parmelia pulverulenta var. Dimidiata Arn., Flora 48: 594. 1864.

Ecology and Distribution: On tree bark, Tikaritola, Near Narmada temple, $1060 \mathrm{~m}$.

Range of Distribution: India (Himachal Pradesh, Karnataka, Madhya Pradesh and Sikkim); Europe, Southern hemisphere.

Specimens examined: Tikaritola, A. N. Shukla 2835, 2877 (BSA); Near Nermada temple, A. N. Shukla 1493 (BSA), det. A. N. Shukla.

71. Physcia tribacia (Ach.) Nyl., Flora 57: 307. 1874;
D. D. Awasthi, Beih. Nova Hedwigia 17: 101. 1965. Lecanora tribacia Ach., Lich. Univ.: 415. 1810.

Ecology and Distribution: On tree bark and rocks, Sambhudhara, $1002 \mathrm{~m}$.

Range of Distribution: India (Himachal Pradesh, Karnataka, Madhya Pradesh, Sikkim, Tamil Nadu and Uttarakhand), Australia, Japan, Nepal; East Africa, Europe, North America.

Specimen examined: Sambhudhara, G. P. Sinha 2732 (BSA), det. A. N. Shukla.

72. Pyxine cocoes (Sw.) Nyl., Mem. Soc. Imp. Sci. Nat. Cherbourgh 5: 108. 1857; D. D. Awasthi, Phytotmorphology 30: 367. 1980. Lichen cocoes Sw., Nov. Gen. Sp. Pl.: 146. 1788.

Ecology and Distribution: On tree bark, Amarkantak helipad area, $1060 \mathrm{~m}$.

Range of Distribution: India (Goa, Karnataka, Kerala, Madhya Pradesh, Maharashtra, Manipur, Orissa, Tamil Nadu, Uttar Pradesh and West Bengal), Sri Lanka; widely distributed in tropical and subtropical regions of the world.

Specimen examined: Amarkantak helipad area, G. P. Sinha 1209 (BSA), det. A. N. Shukla.

73. Pyxine meissnerina Nyl., Bull. Soc. Linn. Normandie, ser. 2, 7: 164. 1973; D. D. Awasthi, Beih. Nova Hedwigia 17: 108. 1965.

Ecology and Distribution: On tree bark, Near Narmada temple, $1060 \mathrm{~m}$.

Range of Distribution: India (Madhya Pradesh, Manipur, Tamil Nadu, Uttar Pradesh, Uttarakhand and West Bengal), Japan, Nepal; Africa, South East Asia.

Specimens examined: Near Nermada temple, A. N. Shukla 1479, 1492 (BSA), det. A. N. Shukla.

74. Pyxine sorediata (Ach.) Mont. in Sagra, Hist. Cuba Bot. 9: 188. 1824; D. D. Awasthi, Beih. Nova Hedwigia 17: 108. 1965. Lecidea sorediata Ach., Syn. Lich.: 54. 1814.

Ecology and Distribution: On tree bark and rocks, Mai-Ki-Bagia, $960 \mathrm{~m}$.

Range of Distribution: India (Arunachal Pradesh, Himachal Pradesh, Karnataka, Kerala, Madhya Pradesh, Manipur, Nagaland, Sikkim, Tamil Nadu, Uttarakhand and West Bengal-hills), Nepal, Sri Lanka, Thailand; widely distributed throughout the world from tropical to subtropical regions.

Specimen examined: Mai-Ki-Bagia, G. P. Sinha 1151 (BSA), det. A. N. Shukla.

75. Pyxine subcinerea Stirt., Trans. \& Proc. New Zealand Inst. 30: 397. 1898; D. D. Awasthi \& Joshi, Geophytology 7(1): 96. 1977.

Ecology and Distribution: On tree bark, Jamunadader, Dhunipani, 900 - $1060 \mathrm{~m}$.

Range of Distribution: Distribution: India (Himachal Pradesh, Jammu \& Kashmir, Madhya Pradesh, Nagaland, 
Sikkim, Tamil Nadu, Uttarakhand and West Bengalhills), Queensland and Thailand.

Specimens examined: Jamunadader, A. N. Shukla 2578 (BSA); Dhunipani, A. N. Shukla 2546 (BSA), det. A. N. Shukla.

\section{Family-Teloschistaceae}

76. Caloplaca citrina (Hoffm.) Th.Fr., Nova Acta Regiae Soc. Sci. Upsal., ser. 3, 3: 218. 1861. Verrucaria citrina Hoffm., Deutschl. Fl.: 198. 1796.

Ecology and Distribution: On tree bark and rocks, Helipad area, $1060 \mathrm{~m}$.

Range of Distribution: India (Jammu \& Kashmir, Madhya Pradesh, Maharashtra and Tamil Nadu), Brazil and moist temperate regions of the world.

Specimen examined: Helipad area, G. P. Sinha 1208 (BSA), det. A. N. Shukla.

77. Caloplaca flavorubescens (Huds.) J. R. Laundon, Lichenologist 8: 147. 1976; D. D. Awasthi, Biblioth. Lichenol. 40: 88. 1991. Lichen flavorubescens Huds., Fl. Angl.: 443. 1762.

Ecology and Distribution: On tree bark and rocks, Jamunadader, $1060 \mathrm{~m}$.

Range of Distribution: India (Himachal Pradesh, Karnataka, Madhya Pradesh, Manipur, Nagaland and Uttarakhand).

Specimen examined: Jamunadader, A. N. Shukla 2591 (BSA), det. A. N. Shukla.

78. Caloplaca vitellinula (Nyl.) H.Oliver, Expos. Lich. Ouest. Fr. 1: 232. 1897; D. D. Awasthi \& Kr.P.Singh, Geophytology 5(1):110. 1975. Lecanora vitellinula Nyl. in Crombie, J. Bot. 13: 334. 1875.

Ecology and Distribution: On rocks, Kapildhara, $1000 \mathrm{~m}$.

Range of Distribution: India (Madhya Pradesh, Manipur and Tamil Nadu), Kerguelenland and temperate areas of Asia and Europe.

Specimen examined: Kapildhara, G. P. Sinha 1194 (BSA), det. A. N. Shukla.

\section{Discussion}

The outcome of this study revealed the report of 78 species of lichens belonging to 25 genera under 9 families, among these Dirinaria consimilis (Stirt.) D. D. Awasthi, Dirinaria applanata (Fée) D. D. Awasthi, Heterodermia albidiflava (Kurok.) D. D. Awasthi, of family Physiaceae, Bulbothrix isidiza (Nyl.) Hale, Parmotrema praesorediosum (Nyl.) Hale, Parmotrema tinctorum (Despr. ex Nyl.) Hale, of family Parmeliaceae, Pertusaria concinna
Erichsen, Pertusaria granulata (Ach.) Müll.Arg., Pertusaria quassiae (Fée) Nyl., of family Pertusariaceae are commonly seen in the biosphere reserve. It is noticed that some localities viz., Sambhudhara, Jamunadader, Karanjia, Panchdhara, Sonemuda and Jagatpur represent rich growth of lichen species and diversity, as well.

\section{Acknowledgements}

The authors are thankful to the Director, Botanical Survey of India, Kolkata for his encouragement and providing the facilities.

\section{REFERENCES}

[1] J. S. Singh and S. P. S. Kushwaha, "Forest Biodiversity and Its Conservation in India," International Forestry Review, Vol. 10, No. 2, 2008, pp. 292-304. doi:10.1505/ifor.10.2.292

[2] A. N. Shukla and K. P. Singh, "Diversity of Woody Plants in Achanakmar-Amarkantak Biosphere Reserve, Central India,” Indian Journal Forestry, Vol. 31, No. 2, 2007, pp. 269-282.

[3] H. G. Champion and S. K. Seth, "A Revised Survey of the Forest Types of India,” Government of India Publication, New Delhi, 1968.

[4] H. O. Saxena, "The Flora of Amarkantak (Madhya Pradesh),” Bulletin of Botanical Survey of India, Vol. 12, 1970, pp. 37-66.

[5] O. P. Mishra, "Addition to the Flora of Amarkantak (M.P.)," Journal of Economic and Taxonomic Botany, Vol. 14, No. 1, 1990, pp. 198-200.

[6] A. N. Shukla, K. P. Singh and A. Kumar, "Ethnoveterinay Uses of Plants from Achanakmar-Amarkantak Biosphere Reserve, Madhya Pradesh and Chhattisgarh,” Journal of Non-Timber Forest Product, Vol. 14, No. 1, 2007, pp. 53-55.

[7] K. P. Singh, A. N. Shukla, S. L. Bondya and S. Mishra, "Flowering Plants of Achanakmar-Amarkantak Biosphere Reserve, Central India,” Journal of Non-Timber Forest Product, Vol. 17, No. 1, 2010, pp. 101-133.

[8] S. Nayaka, Satya and D. K. Upreti, "Lichen Diversity in Achanakmar Wildlife Sanctuary Core Area of proposed Amarkantak Biosphere Reserve, Chhattisgarh,” Journal of Economic and Taxonomic Botany, Vol. 31, No. 1, 2007, pp. 133-142.

[9] D. K. Upreti, S. Nayaka and Satya, "Enumeration of Lichens from Madhya Pradesh and Chhattisgarh, India," Journal of Applied Bioscience, Vol. 31, No. 1, 2005, pp. 55-63. 